\title{
Efficient dual-negative selection for bacterial genome editing
}

\author{
Francesca Romana Cianfanelli ${ }^{\dagger}$, Olivier Cunrath $^{\dagger}$ and Dirk Bumann ${ }^{*}$ (D)
}

\begin{abstract}
Background: Gene editing is key for elucidating gene function. Traditional methods, such as consecutive singlecrossovers, have been widely used to modify bacterial genomes. However, cumbersome cloning and limited efficiency of negative selection often make this method slower than other methods such as recombineering.

Results: Here, we established a time-effective variant of consecutive single-crossovers. This method exploits rapid plasmid construction using Gibson assembly, a convenient E. coli donor strain, and efficient dual-negative selection for improved suicide vector resolution. We used this method to generate in-frame deletions, insertions and point mutations in Salmonella enterica with limited hands-on time. Adapted versions enabled efficient gene editing also in Pseudomonas aeruginosa and multi-drug resistant (MDR) Escherichia coli clinical isolates.

Conclusions: Our method is time-effective and allows facile manipulation of multiple bacterial species including MDR clinical isolates. We anticipate that this method might be broadly applicable to additional bacterial species, including those for which recombineering has been difficult to implement.
\end{abstract}

Keywords: Salmonella, Homologous recombination, Mutagenesis, Gene manipulation, MDR

\section{Background}

Genetic engineering is fundamental for molecular analysis of genotype-phenotype relationships, and for determining the function of previously uncharacterized genes [1-3]. Site-specific mutagenesis can be achieved using different methods. Traditionally, marker-free genetic manipulations were obtained using consecutive single-crossovers mediated by endogenous recombinases $[4,5]$. A suicide vector is first integrated in the desired location using homologous recombination. Bacteria, in which a subsequent second crossover results in loss of the integrated plasmid, can then be selected using counter-selection markers [6-9]. However, counter-selection is often suboptimal resulting in a need to screen many clones for the desired event $[10,11]$. Later, the $\lambda$-Red recombineering technology, a phage-based homologous recombination system based on linear DNA transfer

\footnotetext{
* Correspondence: dirk.bumann@unibas.ch

${ }^{\dagger}$ Francesca Romana Cianfanelli and Olivier Cunrath contributed equally to this work.

Biozentrum, University of Basel, CH-4056 Basel, Switzerland
}

and an exogenous recombinase, was introduced [8, 12-15]. Scarless mutations can be obtained when combining this method with a counter-selection marker [16-19]. Currently, $\lambda$-Red recombineering is the method of choice for introducing genetic manipulations in S. enterica and E. coli [20] but it has been difficult to implement in several other bacterial species such as Pseudomonas aeruginosa. Recently, clustered regularly interspaced short palindromic repeats (CRISPR)-Cas has revolutionized eukaryotic genome editing [21-23], but this strategy is more cumbersome for bacteria with limited recombination activities [24-26].

Here, we combined several improvements for establishing a time-efficient versatile method for consecutive single cross-overs in multiple bacterial species. We used rapid Gibson assembly of PCR products [27] to generate suicide vectors with dual negative selection mediated by I-SceI and SacB [28, 29] (Fig. 1a), which increased counter-selection efficiency to $100 \%$ for nearly all tested deletions, insertions and point mutations. We employed an $E$. coli donor strain that simplifies donor removal 

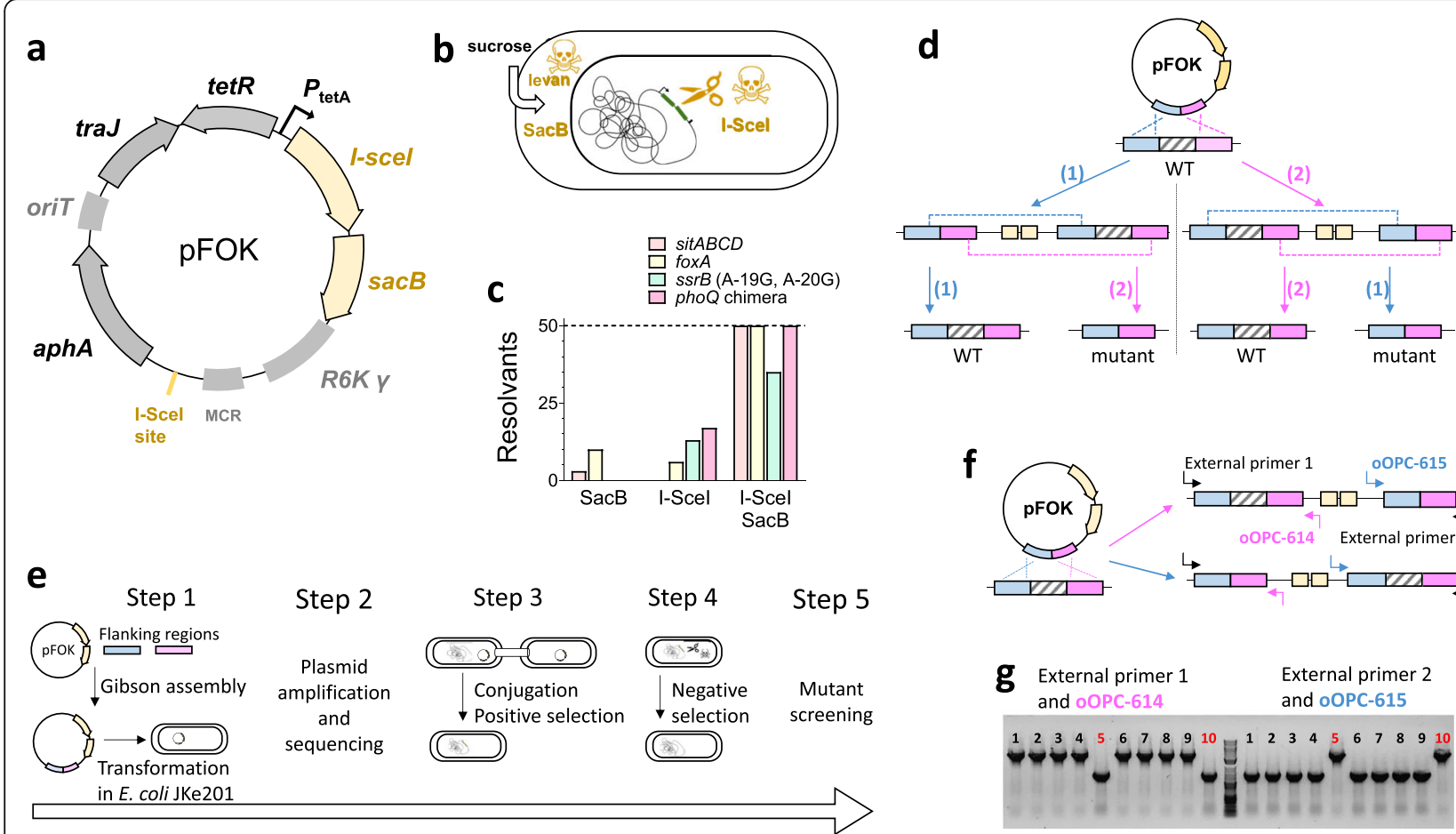

Fig. 1 An optimized method for genome editing in Salmonella enterica. a Map of suicide plasmid pFOK (aphA, aminoglycoside phosphotransferase gene conferring resistance to kanamycin; I-scel gene encoding meganuclease; oriT, origin of conjugational transfer; $P_{\text {tet } A}$, tet $A$ promoter; R6K y ori, pi-dependent origin of replication; $s a c B$, levansucrase gene; tetR, tetracycline repressor gene; traJ, transcriptional activator for conjugational transfer genes; MCR, multi cloning region containing EcoRl and BamHI recognition sites). b Mechanisms of negative selection for $\mathrm{SaCB}$ and I-Scel, c Efficiency of negative selection for various chromosomal loci (sitABCD deletion - orange, foxA deletion - yellow, ssrB point mutation - green, and phoQ chimeric insertion - magenta [30]) using either SacB or I-Scel, or a combination of both. Fifty colonies were screened for each mutation. $\mathbf{d}$ Schematic representation of the consecutive single crossover procedure. Recombination can occur in one of the two homologous sequences (routes 1 and 2). Only alternate single crossover events involving both homologous sequences lead to the desired mutation, while two consecutive single crossovers in the same regions lead to reversion to wild-type (WT) e Overview of the entire procedure. Ideally, each step can be completed in one working day. $\mathbf{f}$ Schematic representation of preferential recombination in the right flanking region. External primers 1 and 2 together with plasmid-specific primers oOPC-614 and OOPC-615 can be used to screen co-integrant clones to reveal such bias and to identify rare variants for promoting mutant generation in the second single crossover. $\mathbf{g}$ Recombination bias for foxA deletion. PCR results of ex-conjugant screening using external primer 1 (oOPC-396) / OOPC-614 or external primer 2 (oOPC-397) / oOPC-615. Rare exconjugants (clones 5,10$)$ with recombination in the non-preferred flanking region were used for subsequent counter-selection

after conjugation and avoids common problems with contaminating phages [31]. We used different positive selection markers that enable selection in many bacterial species, including MDR pathogens [32]. Combination of these elements yielded a reliable and fast method for genetic engineering of multiple bacterial species that, in concert with a simplified screening procedure, minimized hands-on time and significantly accelerated genome editing in our lab.

\section{Results}

Our goal was a rapid and efficient genetic editing method with minimal hands-on time. For this purpose, we combined rapid plasmid construction using Gibson assembly [27], a phage-free, pir-carrying (for propagation of $R 6 \mathrm{K \gamma}$ plasmids), diaminopimelic acid (DAP)-dependent E. coli donor strain JKe201 [31] for plasmid amplification and conjugation, with subsequent facile removal of donor in absence of DAP, and an improved dual-negative counterselection. We generated suicide vectors from PCR fragments with automatically designed primers using Gibson assembly [27]. Each vector carries commonly used genetic elements for conditional propagation ("suicide vector" with pi-dependent replication from $R 6 K \gamma$ ), conjugation (oriT, tra) and selection for two sequential singlecrossovers. For the first positive selection, we used aphA conferring resistance to kanamycin (pFOK, Fig. 1a).

A major limitation to efficient genetic editing using two consecutive single-crossovers has been inefficient counter-selection of the second recombination, in part due to inactivating mutations in the negative selection marker [33]. We tested counter-selection efficiency in multiple Salmonella loci using the commonly used markers $s a c B$ or I-sceI (Fig. 1b). sacB codes for levan sucrase, which confers sensitivity to sucrose because of accumulation of the toxic product levan in the periplasm 
[28]. I-sceI codes for the restriction enzyme I-SceI, which causes lethal DNA double-strand breaks when a I-SceI recognition sequence is present on the genome [29]. To assess counter-selection efficiency of SacB or I-SceI singly, we generated plasmid variants (pOPC-001 and pOPC-003) differing just in the counter-selection. Counter-selection was suboptimal for both markers with marker-free clones representing none or only a minority of the recovered colonies (Fig. 1c). Consequently, many colonies had to be tested for finding the desired clones. To overcome this problem, we generated a new suicide vector, pFOK, combining both $s a c B$ and $I$-sceI under the regulatory control of the TetR regulator (Fig. 1a). We tested the TetR system using the green fluorescent protein (GFP) as reporter on the same pSC101 backbone and found no detectable GFP fluorescence above the autofluorescence background in absence of the inducer anhydro-tetracycline indicating limited leakiness in our conditions (Supplementary Fig. S1). Cells carrying the conditional dual-negative selection cassette under control of the TetR system showed no decrease in cloning efficiency but efficient negative selection in presence of sucrose and anhydro-tetracycline, yielding only, or a large majority, of resolvants that had successfully cured pFOK from their chromosome (Fig. 1c). A similar dual-negative selection has been previously described for Gram-positive bacteria [34]. The ratios for the two alternative results - mutation or reversion back to wild-type - varied between the individual mutants (Supplementary Fig. S2).

To expand our gene manipulation method to other bacterial species, including those for which $\lambda$-Red recombineering has not yet been established, we used alternative positive selection markers. This included aac (3)-I, coding for a aminoglycoside $\mathrm{N}$-acetyltransferase that confers resistance to gentamicin which can be used as an alternative in bacteria, including Pseudomonas aeruginosa, which are resistant to kanamycin but susceptible to gentamicin (pFOG,
Fig. 2a). We confirmed the utility of pFOG by deleting the mex $A B$ operon in $P$. aeruginosa and observed 50 resolvants among 50 tested colonies (100\%) after negative selection. As an alternative, we combined $a p h A$ with a second positive marker, tpm, yielding suicide vector pFOKT (Fig. 2b). tpm codes for a thiopurine-S-methyltranferase conferring resistance to tellurite [35]. This plasmid can be used for multi-drug resistant (MDR) bacteria for which the choice of positive selection markers is limited [32]. To limit toxic exposure to volatile dimethyl telluride, we used kanamycin for suicide vector generation and used tellurite only for the positive selection of ex-conjugants. We confirmed the utility of pFOKT by deleting tolC with high efficiency in a multi-drug resistant clinical Escherichia coli isolate [32] and again observed 50 resolvants among 50 tested colonies (100\%) after negative selection.

In some cases, the second single-crossover had a high bias for resolution to wild-type loci (instead of the desired mutant). This was usually due to differences in recombination frequency between the two flanking regions. PCR primers (oOPC-614 and oOPC-615) that bind in the plasmid, combined with chromosomal primers outside the flanking regions in the merodiploids (Fig. 1f, g), enabled detection of such biases. For these cases, we selected exconjugants in which the first single-crossover had occurred in the non-preferred flanking region. In these clones, we often observed frequent resolution to mutant loci during the second single-crossover (deletion of foxA, Supplementary Fig. S2).

Altogether, the whole protocol from initial plasmid construction to scar-less sequence-verified mutant strains (Fig. 1e) was completed within five working days with minimal hands-on time for 23 of 30 Salmonella mutants. We obtained all residual mutants after additional optimization of the initial PCR (four mutants), prolonged cultivation times for mutants with reduced growth (two mutants), or screening for biased recombination as shown
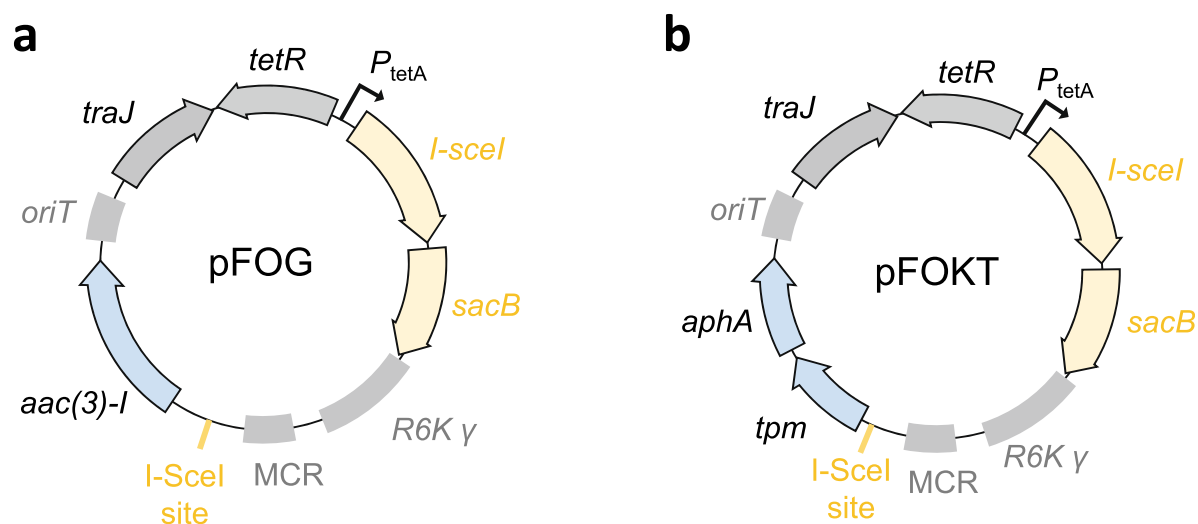

Fig. 2 Maps of alternative suicide plasmids. a pFOG carrying aac (3)-I which confers resistance to gentamicin. b pFOKT carrying tpm coding for thiopurine-S-methyltranferase which confers resistance to tellurite 
in Fig. 1f, g (one mutant $-\triangle f o x A$ ). The Escherichia tolC mutant was also completed within 5 working days, while slow growth of Pseudomonas at $28^{\circ} \mathrm{C}$ during the negative selection required a total of 6 working days. In all cases, we obtained $100 \%$ resolvants after a single round of negative selection confirming the efficiency of our method (the only exception was the $s s r B$ point mutant with $70 \%$ resolvants as shown in Fig. 1c).

\section{Discussion}

Gene editing enables investigation of gene function. Here, we improved on a widely used method of consecutive single-crossovers. Our newly developed suicide vectors, based on a highly efficient dual negative selection strategy, mitigate the major pitfall of consecutive singlecrossovers: the poor selection of resolvant clones after the second recombination. Thus, our new vectors do not require multiple rounds of counter-selection to ensure resolution of the suicide vector from the recipient strain. One of the two negative selection genes encodes I-SceI which recognizes a specific 18-basepair sequence [36]. While none of the strains used in this study harbored a I-SceI recognition sequence in their genomes, this sequence might be present in other bacteria which would need method adaptation. Gibson assembly enables rapid construction of plasmids with PCR fragments with no need for enzyme digestion and ligation, and no sequence constraints due to restriction sites. Our approach relies on endogenous RecA, but not the heterologous, powerful lambda-red recombinase, which might minimize the risk of secondary mutations. Purifying mutated loci by generalized phage transduction may thus not be required. Our method employs conjugation instead of electroporation (as required for lambda-red methods), which minimizes culture volumes and hands-on time. We anticipate that this method might be broadly applicable to additional bacterial species, including those for which recombineering has been difficult to implement.

\section{Conclusions}

Our plasmids and protocols provide facile time-efficient methods for genetic engineering in multiple bacterial species including MDR clinical isolates.

\section{Methods}

\section{Media and strains}

Bacterial strains were cultured in Lennox lysogeny broth (LB) (tryptone $10 \mathrm{~g} / \mathrm{L}$, yeast extract $5 \mathrm{~g} / \mathrm{L}$ and $\mathrm{NaCl} 5 \mathrm{~g} /$ L) medium. E. coli JKe201 [31] was cultured in the presence of $100 \mu \mathrm{M}$ of diamino pimelic acid (DAP) (Sigma Aldrich D1377-10G). Salmonella enterica serovar Typhimurium SL1344 was cultured in LB in the presence of $90 \mu \mathrm{g} / \mathrm{ml}$ streptomycin (Sigma-Aldrich S9137-100G). E. coli EC01 [32] and P. aeruginosa UCBPP-PA14 were cultured in LB. For preparing chemically competent cells, fresh LB medium was inoculated at $\mathrm{OD}_{600 \mathrm{~nm}} 0.01$ with an overnight culture of JKe201 and grown until $\mathrm{OD}_{600 \mathrm{~nm}}$ 0.4-0.6. Bacteria were washed twice with $25 \mathrm{ml}$ of ice-cold $100 \mathrm{mM} \mathrm{CaCl}_{2}$ (Sigma Aldrich C1016-500G) solution containing $15 \%$ of glycerol (AppliChem, A1123, 1000). Bacteria were resuspended in $5 \mathrm{ml}$ ice-cold $\mathrm{CaCl}_{2}$ $100 \mathrm{mM} / 15 \%$ glycerol and $200 \mu \mathrm{l}$ aliquots were frozen and stored at $-80^{\circ} \mathrm{C}$. Super-Optimal broth with Catabolite repression (SOC) (tryptone $20 \mathrm{~g} / \mathrm{L}$, yeast extract 5 $\mathrm{g} / \mathrm{L}, \mathrm{NaCl} 0.5 \mathrm{~g} / \mathrm{L}, \mathrm{KCl} 0.186 \mathrm{~g} / \mathrm{L}, \mathrm{MgSO}_{4} 4.8 \mathrm{~g} / \mathrm{L}$ and glucose $3.6 \mathrm{~g} / \mathrm{L}$ ) medium was used for resuspension after heat shock. $50 \mu \mathrm{g} / \mathrm{ml}$ kanamycin (Roth T832.4) or $15 \mu \mathrm{g} /$ $\mathrm{ml}$ gentamicin (Gibco 15,750-037) were used to select E. coli transformants. For positive selection, kanamycin (Roth T832.4) at a final concentration of $50 \mu \mathrm{g} / \mathrm{ml}$, gentamicin (Gibco 15,750-037) at a final concentration of $30 \mu \mathrm{g} / \mathrm{ml}$, or potassium tellurite (Sigma P0677) at a final concentration of $10 \mu \mathrm{g} / \mathrm{ml}$, were used. Counter-selection plates contained LB-no salt $(10 \mathrm{~g} / \mathrm{L}$ tryptone, $5 \mathrm{~g} / \mathrm{L}$ yeast extract), 20\% (w/v) sucrose (Sigma-Aldrich 84,097-1KG), $15 \mathrm{~g} / \mathrm{L}$ agar and $0.5 \mu \mathrm{g} / \mathrm{ml}$ anhydrous tetracycline (AHT) (Sigma-Aldrich 37,919-100MG-R).

\section{Generation of the suicide vectors}

Primers for generating pOPC-001, pOPC-003 and pFOK are reported in Supplementary Table S1. pOPC-001 was obtained by combining the kanamycin resistance cassette and the I-SceI restriction site from pWRG717 [37], the origin of replication $(\mathrm{R} 6 \mathrm{~K} \gamma)$ and origin of transfer (oriT) from pGP704 [6, 38] and the tetR and I-sceI locus from pWRG730 [37] using Gibson assembly. pOPC-003 was generated by replacing the tet $R$ and I-sceI locus from pOPC-001 with $s a c B$ from pEXG2 [39]. pFOK (5841 bp) was generated by inserting $s a c B$ amplified from pOPC-003 downstream of the I-sceI gene on pOPC-001. pFOG (5659 bp) was generated by replacing aphA of pFOK by acc (3)-I. pFOKT (6668 bp) was generated by insertion of tpm [35] between aphA and the multi cloning region (MCR).

\section{Amplification of the upstream and downstream regions}

Flanking primers with a $40 \mathrm{bp}$ overlap were designed to amplify $700 \mathrm{bp}$ up- and downstream of the gene of interest using SnapGene ${ }^{\circ}$ (version 4.0.3) with the Gibson Assembly tool (Supplementary Table S1). Fragments were amplified using a high-fidelity polymerase mix (KOD Hot Start Master Mix, Millipore) and separated on a $1 \%$ agarose gel. Vectors were purified from overnight cultures using a plasmid miniprep kit ZymoPURE $^{\mathrm{Tw}}$, ZymoResearch). Vectors were digested using EcoRI-HF and BamHI-HF (New England BioLabs) for $1 \mathrm{~h}$ at $37^{\circ} \mathrm{C}$, or PCR-amplified, and purified on agarose gel. Alternatively, vectors can also be amplified by long-range PCR. Final concentrations of amplificated fragments and 
digested vectors were measured using a microvolume spectrometer $\left(\right.$ Colibri $\left.^{\circledR}\right)$.

\section{Gibson assembly and chemical transformation}

Plasmids generated in this study are listed in Supplementary Table S2. Gibson assembly reaction was performed as described [27]. The reaction mix contained $50 \mathrm{ng}$ of each upand downstream fragments and $150 \mathrm{ng}$ of suicide vector, and Gibson assembly mix 1x (New England BioLabs) in a total volume of $10 \mu \mathrm{l}$. The reaction mixture was incubated at $50{ }^{\circ} \mathrm{C}$ for $20 \mathrm{~min}$. Five microliters of the reaction mixture was added to a $100 \mu \mathrm{l}$ aliquot of $E$. coli JKe201 heat-shock competent bacteria and incubated for $30 \mathrm{~min}$ on ice. After a heat shock at $42^{\circ} \mathrm{C}$ for $30 \mathrm{~s}$ followed by $2 \mathrm{~min}$ on ice, bacteria were resuspended in $1 \mathrm{ml}$ prewarmed SOC medium containing $100 \mu \mathrm{M}$ of DAP and incubated for $1 \mathrm{~h}$ at $37^{\circ} \mathrm{C}$. Transformants were selected on Lennox-LB agar plates containing $100 \mu \mathrm{M}$ DAP (required for growth of JKe201) and either $50 \mu \mathrm{g} / \mathrm{ml}$ kanamycin or $15 \mu \mathrm{g} / \mathrm{ml}$ gentamicin. Clones were screened using PCR with primers oOPC-614 and oOPC-615 (Supplementary Table S1).

\section{Conjugation and selection of the first homologous recombination event}

The recipient $S$. Typhimurium and $E$. coli strains were inoculated in $2 \mathrm{ml}$ of LB containing no antibiotics at $37^{\circ} \mathrm{C}$. $P$. aeruginosa was inoculated in $2 \mathrm{ml} \mathrm{LB}$ without antibiotics at $42^{\circ} \mathrm{C}$. The donor $E$. coli strain was inoculated in 2 $\mathrm{ml}$ of LB containing $100 \mu \mathrm{M}$ DAP but no antibiotics. Five hundred microliters each of overnight cultures of the donor $E$. coli strain and the recipient strain were washed with LB, mixed and centrifuged together. The pellet was resuspended in $50 \mu \mathrm{LB}$ and deposited on $22 \mathrm{~mm}$ filter membranes with $0.45 \mu \mathrm{m}$ pores (Millipore, Merck) on a pre-dried LB agar plate. After mating for $6 \mathrm{~h}$ at $37^{\circ} \mathrm{C}$, bacteria were scraped from the filter membrane and resuspended in $1 \mathrm{ml}$ LB. Merodiploid $S$. Typhimurium (pFOK) were selected on LB plates containing $90 \mu \mathrm{g} / \mathrm{ml}$ streptomycin and $50 \mu \mathrm{g} / \mathrm{ml}$ kanamycin at $37^{\circ} \mathrm{C}$ for at least $16 \mathrm{~h}$. E. coli (pFOKT) and $P$. aeruginosa (pFOG) merodiploids were selected on LB plates containing $10 \mu \mathrm{g} / \mathrm{ml}$ tellurite or $30 \mu \mathrm{g} / \mathrm{ml}$ gentamicin, respectively. Clones grew on tellurite to form black colonies.

\section{Counter-selection of the second homologous recombination event}

At least three trans-conjugant colonies were combined and grown for $4 \mathrm{~h}$ at $37^{\circ} \mathrm{C}$ in $2 \mathrm{ml}$ of LB. Bacteria were then streaked on freshly prepared LB-no salt agar plates [24] containing 20\% sucrose and $0.5 \mu \mathrm{g} / \mathrm{ml} \mathrm{AHT}$. Plates were incubated at $28{ }^{\circ} \mathrm{C}$ protected from light for at least $24 \mathrm{~h}$. Colonies were screened for the desired mutation using PCR with external primers (Supplementary Table S1). Mutants were confirmed by DNA-sequencing (Microsynth.ch).

\section{Supplementary information}

Supplementary information accompanies this paper at https://doi.org/10. 1186/s12866-020-01819-2.

Additional file 1: Figure S1. Activity of the TetR system regulating expression of the green fluorescent protein (GFP) in absence and presence of its inducer anhydro-tetracycline (aTC) as measured by flow cytometry (AF, autofluorescence of a strain without $g f p$ ).

Additional file 2: Figure S2. Resolution results (mutant or reversion back to wild-type) for 50 colonies obtained after negative selection. The results for deletion of foxA were obtained from clone 5 shown in Fig. $1 \mathrm{~g}$.

Additional file 3: Table S1. Primers used in this study. Table S2. Plasmid used in this study.

\section{Abbreviations}

AHT: Anhydro-tetracycline; CRISPR: Clustered regularly interspaced short palindromic repeats; DAP: Diaminopimelic acid; GFP: Green fluorescent protein; MDR: Multi-drug resistant; LB: Lysogeny broth; OD: Optical density; SOC: Super-Optimal broth with Catabolite repression

\section{Acknowledgements}

We thank all group members for their helpful feedback.

\section{Authors' contributions}

D.B. designed the study with input from F.R.C and O.C.; F.R.C. and O.C. constructed plasmids and mutants; F.R.C. and O.C. wrote the manuscript with early input from D.B. and subsequently all authors approved the final manuscript.

Authors' information

F.R.C and O.C. contributed equally to this work.

\section{Funding}

This study was supported in part by grants from the Swiss National Foundation (310030_156818 to D.B.). The funding had no influence in the design of the study, the collection, the analysis, the interpretation of data and the writing of the manuscript.

Availability of data and materials

All data generated or analysed during this study are included in this published article.

Ethics approval and consent to participate

Not applicable.

\section{Consent for publication}

Not applicable.

\section{Competing interests}

The authors declare that they have no competing interests.

Received: 25 March 2020 Accepted: 11 May 2020

Published online: 24 May 2020

References

1. Court DL, Sawitzke JA, Thomason LC. Genetic engineering using homologous recombination. Annu Rev Genet. 2002;36:361-88.

2. Camps M, Loeb LA. Targeted mutagenesis in E. coli: a powerful tool for the generation of random mutant libraries. Discov Med. 2003;3(18):36-7.

3. Tagini F, Greub G. Bacterial genome sequencing in clinical microbiology: a pathogen-oriented review. Eur J Clin Microbiol Infect Dis. 2017;36(11):2007-20.

4. Link AJ, Phillips D, Church GM. Methods for generating precise deletions and insertions in the genome of wild-type Escherichia coli: application to open reading frame characterization. J Bacteriol. 1997;179(20):6228-37.

5. Hmelo LR, et al. Precision-engineering the Pseudomonas aeruginosa genome with two-step allelic exchange. Nat Protoc. 2015;10(11):1820-41.

6. Miller VL, Mekalanos JJ. A novel suicide vector and its use in construction of insertion mutations: osmoregulation of outer membrane proteins and 
virulence determinants in Vibrio cholerae requires toxR. J Bacteriol. 1988 170(6):2575-83.

7. Blomfield IC, et al. Allelic exchange in Escherichia coli using the Bacillus subtilis sacB gene and a temperature-sensitive pSC101 replicon. Mol Microbiol. 1991;5(6):1447-57.

8. Zhang N, et al. I-Scel-mediated scarless gene modification via allelic exchange in clostridium. J Microbiol Methods. 2015;108:49-60.

9. Reyrat JM, et al. Counterselectable markers: untapped tools for bacterial genetics and pathogenesis. Infect Immun. 1998;66(9):4011-7.

10. Wu SS, Kaiser D. Markerless deletions of pil genes in Myxococcus xanthus generated by counterselection with the Bacillus subtilis sacB gene. J Bacteriol. 1996;178(19):5817-21.

11. Ishikawa M, et al. Efficient counterselection for Methylococcus capsulatus (Bath) by using a mutated pheS gene. Appl Environ Microbiol. 2018;84(23) https://doi.org/10.1128/AEM.01875-18.

12. Datsenko KA, Wanner BL. One-step inactivation of chromosomal genes in Escherichia coli K-12 using PCR products. Proc Natl Acad Sci U S A. 2000; 97(12):6640-5.

13. Derbise A, et al. A rapid and simple method for inactivating chromosomal genes in Yersinia. FEMS Immunol Med Microbiol. 2003;38(2):113-6.

14. Murphy KC, Campellone KG, Poteete AR. PCR-mediated gene replacement in Escherichia coli. Gene. 2000;246(1-2):321-30.

15. Karlinsey JE. lambda-Red genetic engineering in Salmonella enterica serovar Typhimurium. Methods Enzymol. 2007;421:199-209.

16. Cox MM, et al. Scarless and site-directed mutagenesis in Salmonella enteritidis chromosome. BMC Biotechnol. 2007;7:59.

17. Blank K, Hensel M, Gerlach RG. Rapid and highly efficient method for scarless mutagenesis within the Salmonella enterica chromosome. PLoS One. 2011;6(1):e15763.

18. Li XT, et al. Positive and negative selection using the tetA-sacB cassette: recombineering and P1 transduction in Escherichia coli. Nucleic Acids Res. 2013:41(22):e204.

19. Stringer AM, et al. FRUIT, a scar-free system for targeted chromosomal mutagenesis, epitope tagging, and promoter replacement in Escherichia coli and Salmonella enterica. PLoS One. 2012;7(9):e44841.

20. Kim J, et al. A versatile and highly efficient method for scarless genome editing in Escherichia coli and Salmonella enterica. BMC Biotechnol. 2014;14:84.

21. Cong $L$, et al. Multiplex genome engineering using CRISPR/Cas systems. Science. 2013;339(6121):819-23.

22. Jiang $W$, et al. RNA-guided editing of bacterial genomes using CRISPR-Cas systems. Nat Biotechnol. 2013;31(3):233-9.

23. Adli M. The CRISPR tool kit for genome editing and beyond. Nat Commun. 2018;9(1):1911

24. Pyne ME, et al. Coupling the CRISPR/Cas9 system with lambda red Recombineering enables simplified chromosomal gene replacement in Escherichia coli. Appl Environ Microbiol. 2015;81(15):5103-14.

25. Zhang $\mathrm{H}$, et al. A novel and efficient method for bacteria genome editing employing both CRISPR/Cas9 and an antibiotic resistance cassette. Front Microbiol. 2017:8:812.

26. Ramachandran G, Bikard D. Editing the microbiome the CRISPR way. Philos Trans R Soc Lond Ser B Biol Sci. 2019;374(1772):20180103.

27. Gibson DG, et al. Enzymatic assembly of DNA molecules up to several hundred kilobases. Nat Methods. 2009:6(5):343-5.

28. Steinmetz $\mathrm{M}$, et al. Genetic analysis of sacB, the structural gene of a secreted enzyme, levansucrase of Bacillus subtilis Marburg. Mol Gen Genet. 1983;191(1):138-44.

29. Posfai $\mathrm{G}$, et al. Markerless gene replacement in Escherichia coli stimulated by a double-strand break in the chromosome. Nucleic Acids Res. 1999; 27(22):4409-15.

30. Prost $L R$, et al. The PhoQ histidine kinases of Salmonella and Pseudomonas spp. are structurally and functionally different: evidence that $\mathrm{pH}$ and antimicrobial peptide sensing contribute to mammalian pathogenesis. Mol Microbiol. 2008;69(2):503-19.

31. Harms A, et al. A bacterial toxin-antitoxin module is the origin of inter-bacteria and inter-kingdom effectors of Bartonella. PLoS Genet. 2017;13(10):e1007077.

32. Cunrath $\mathrm{O}$, et al. Quantitative contribution of efflux to multi-drug resistance of clinical Escherichia coli and Pseudomonas aeruginosa strains. EBioMedicine. 2019;41:479-87.

33. Wang $T$, et al. An update of the suicide plasmid-mediated genome editing system in Corynebacterium glutamicum. Microb Biotechnol. 2019;12(5):907-19.
34. Ma W, et al. Development of a markerless gene replacement system in Corynebacterium glutamicum using upp as a counter-selection marker. Biotechnol Lett. 2015;37(3):609-17.

35. Sanchez-Romero JM, Diaz-Orejas R, De Lorenzo V. Resistance to tellurite as a selection marker for genetic manipulations of Pseudomonas strains. Appl Environ Microbiol. 1998;64(10):4040-6.

36. Bellaiche Y, Mogila V, Perrimon N. I-Scel endonuclease, a new tool for studying DNA double-strand break repair mechanisms in Drosophila. Genetics. 1999;152(3):1037-44.

37. Hoffmann $\mathrm{S}$, et al. Scarless deletion of up to seven methyl-accepting chemotaxis genes with an optimized method highlights key function of CheM in Salmonella Typhimurium. PLoS One. 2017;12(2):e0172630.

38. Kolter R, Inuzuka M, Helinski DR. Trans-complementation-dependent replication of a low molecular weight origin fragment from plasmid R6K. Cell. 1978;15(4):1199-208.

39. Rietsch A, et al. ExsE, a secreted regulator of type III secretion genes in Pseudomonas aeruginosa. Proc Natl Acad Sci U S A. 2005;102(22):8006-11.

\section{Publisher's Note}

Springer Nature remains neutral with regard to jurisdictional claims in published maps and institutional affiliations.

Ready to submit your research? Choose BMC and benefit from:

- fast, convenient online submission

- thorough peer review by experienced researchers in your field

- rapid publication on acceptance

- support for research data, including large and complex data types

- gold Open Access which fosters wider collaboration and increased citations

- maximum visibility for your research: over $100 \mathrm{M}$ website views per year

At BMC, research is always in progress.

Learn more biomedcentral.com/submissions 